Research Paper

\title{
Simplified Stereological Evaluation of Renal Morphology after Unilateral Ureteral Obstruction
}

\author{
Peng $\mathrm{Hu}^{1}{ }^{\bowtie}$, Xue Qi Zhao ${ }^{1}$, Jing Wang${ }^{1}$, Jie Cai ${ }^{1}$, Bo Hu${ }^{1}$, Ling Lu${ }^{1}$, Qiang Xuan², Yuan Han Qin ${ }^{3}$ \\ 1. Department of Pediatrics, the First Affiliated Hospital of Anhui Medical University, No. 218 Ji-Xi Road, Hefei 230022, PR China; \\ 2. Department of Urology, Anhui Provincial Hospital, Anhui Medical University, No. 17 Lu-Jiang Road, Hefei 230001, PR China; \\ 3. Department of Pediatrics, the First Affiliated Hospital of Guangxi Medical University, No. 6 Shuang-Yong Road, Nanning 530021, PR \\ China.
}

$\triangle$ Corresponding author: Tel.: +86 551 2922058; E-mail: hupeng28@yahoo.com.cn (P. Hu).

() Ivyspring International Publisher. This is an open-access article distributed under the terms of the Creative Commons License (http://creativecommons.org/ licenses/by-nc-nd/3.0/). Reproduction is permitted for personal, noncommercial use, provided that the article is in whole, unmodified, and properly cited.

Received: 2012.09.07; Accepted: 2013.01.23; Published: 2013.02.21

\begin{abstract}
Objective and methods: This study established a simple stereological method to obtain quantitative information about two- or three-dimensional structures based on observations from kidney sections in the unilateral ureteral obstruction(UUO) model. Results: Tubulointerstitial area(TA) and TA/the area of a rectangular field(RA) were raised gradually, but significantly, in the obstructed kidney from I to 3months post-ligation in comparison to the sham kidney of sham-operated rats(SOR). On the contrary, glomerular area(GA) and glomerular volume(GV) were decreased progressively over time, but significantly, in the obstructed kidney from 3 weeks to 3 months post-ligation compared to the sham kidney of SOR. UUO caused a progressive decline of TA and TA/RA in the contralateral kidney. More specifically, there were significant decreases in TA at I,2,3months post-ligation, while in TA/RA only at 3months post-ligation in comparison to the right kidney of SOR. In contrast, GA and GV enhanced in a time-dependent manner in the contralateral kidney, in which the difference in GA reached significance only at 3months post-ligation, whereas the difference in GV reached significance from I to 3months post-ligation when comparing with the right kidney of SOR. Conclusions: Our results confirmed two typical features of obstructive nephropathy, including widen interstitial space and glomerular atrophy in the obstructed kidney, and compensatory growth of the contralateral kidney.
\end{abstract}

Key words: Compensatory hypertrophy; kidney section; stereological method; unilateral ureteral obstruction; Wistar rat.

\section{Introduction}

Obstructive nephropathy is a common cause of renal insufficiency in children and adults. Unilateral ureteral obstruction (UUO) is a well-established model known to imitate the process of obstructive nephropathy in a simple, accelerated and species-independent manner [1]. Previous studies from our laboratory and others demonstrate that the morphological lesions in the obstructed kidney are characterized by peritubular capillaries rarefaction, tubular atrophy, inflammatory infiltration, widen interstitial space, and progressive tubulointerstitial fibrosis
$[2,3]$. In addition, compensatory hypertrophy of the contralateral kidney is another typical feature in the UUO model [4]. These recognitions of obstructive nephropathy derive mainly from microscope observation or semi-quantitative analysis of kidney sections.

Since 1980, several stereological methods have been established to obtain quantitative information about two- or three-dimensional structures based on observations from kidney sections [5]. The greatest advantage of stereological evaluation is that it can be 
used to minimize the workload in sampling while still providing reliable, quantitative information about the whole structure of interest. However, the problem that confronts the researcher is that a single kidney structure can generate widely differing sections or projections, and several different kidney structures may generate similar sections or projections [6]. Moreover, the tedious and unintelligible methemetical reasoning also severely restricts its clinical application. Given this background, a more simple stereological strategy was applied in the present study to investigate tubulointerstitial area (TA), TA / the area of a rectangular field (RA), glomerular area (GA) and glomerular volume (GV) in both the obstructed kidney and the contralateral kidney.

\section{Materials and methods}

\section{Animals and treatment}

Male Wistar rats weighting 190 to $250 \mathrm{~g}$ were used in the present study. All animal experimentation was performed at the animal facility within the Preclinical Medicine Institute of Anhui Medical University. The procedures and protocols were approved by the Institutional Animal Care and Use Committee. 96 rats were separated into 16 experimental groups: 8 groups undergoing left proximal unilateral ureteral obstruction (UUO) $(n=6)$ and 8 groups with sham-operated rats (SOR) $(n=6)$. All animals were operated under intraperitoneal pentobarbital anesthesia $(60 \mathrm{mg} / \mathrm{kg}$ body weight) and sterile conditions. UUO rats underwent left proximal ureteral ligation with 4-0 silk at the junction of the upper with the two lower thirds of its length. The ureter was cut between

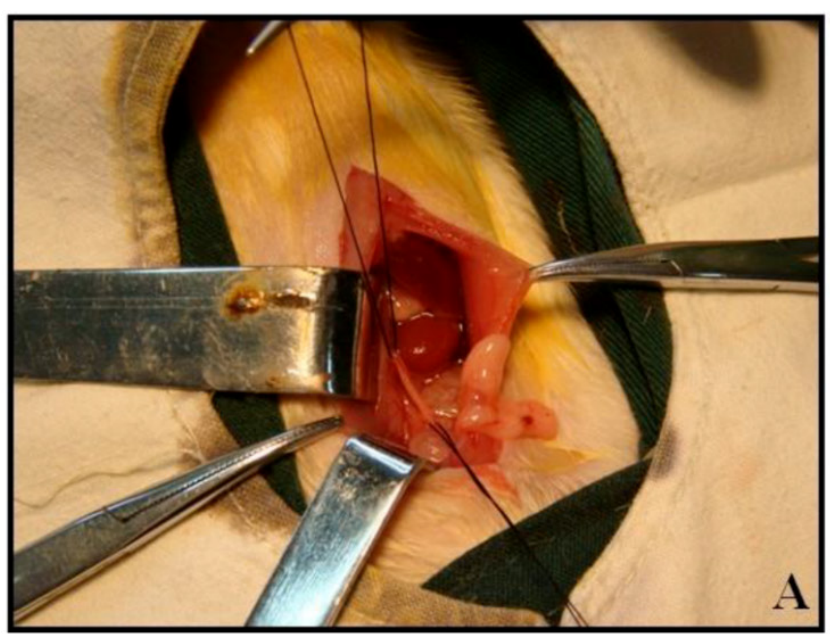

Fig. I. UUO establishment and kidney harvest at $3 \mathrm{~m}$ post-ligation. the ligatures to prevent retrograde urinary tract infection (Fig. 1A). SOR underwent a sham laparotomy with ureteric manipulation through a midline incision. No antibiotics were given. Animals were anesthetized by intraperitoneal pentobarbital injection and sacrificed by heart puncture at $24 \mathrm{~h}, 72 \mathrm{~h}, 1 \mathrm{w}, 2 \mathrm{w}, 3 \mathrm{w}$, $1 \mathrm{~m}, 2 \mathrm{~m}$ and $3 \mathrm{~m}$ post-ligation, respectively. Bilateral kidneys of UUO rats and SOR were harvested (Fig. 1B).

\section{Renal morphology}

At harvest, each kidney was washed with saline, blotted dry on gauze, and weighed. Midcoronal kidney sections were fixed in $4 \%$ paraformaldehyde and embedded in paraffin. Paraffin sections (4 $\mu \mathrm{m}$ thick) were stained with hematoxylin and eosin, and examined independently by two pathologists blinded to the experimental design.

\section{Stereological evaluation}

Stereological evaluation was determined in each section selected at random, and 10 rectangular fields were examined under $\times 400$ magnification. $G A=\pi r_{1}^{2}+$ $\Pi r_{2}{ }^{2}+\Pi r_{3}{ }^{2}+\ldots+\Pi r_{n}{ }^{2}$. The radius of each glomerulus determined by halving the diameter, which would be easier to measure in the profile rather than trying to estimate its center point. TA can be estimated by subtracting total GA from RA, TA = RA - GA (Fig. 2A).

Renal glomerulus is a spherical capillary network. When an entire glomerulus is cut into different sections, the maximum radius $\left(r_{\max }\right)$ is very close to the actual radius of glomerulus, $G V=4 \Pi r_{\max ^{3}} / 3$ (Fig. 2B).

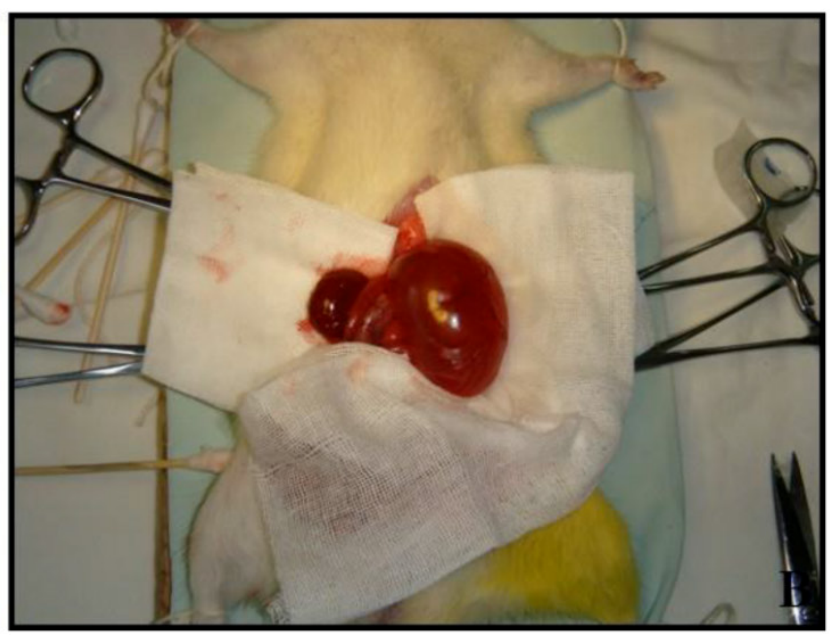



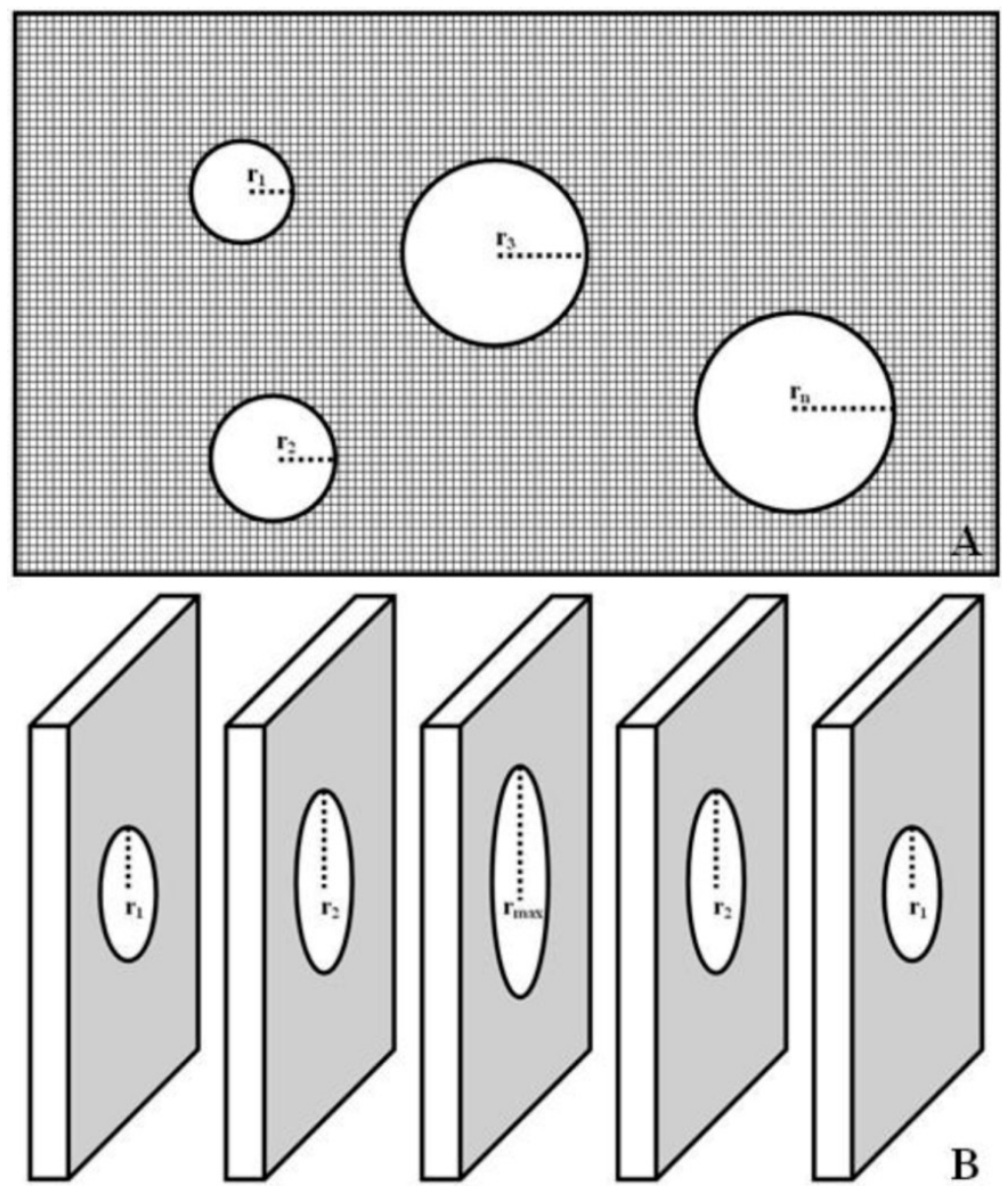

Fig. 2. Stereological evaluation of TIP and GV. TA $=R A-\left(\pi r_{1}{ }^{2}+\pi r_{2}{ }^{2}+\pi r_{3}{ }^{2}+\ldots+\pi r_{n}{ }^{2}\right)(F i g . ~ 2 A) ; G V=4 \pi r_{\max }{ }^{3} / 3(F i g .2 B)$.

\section{Statistical Analyses}

All values are expressed as mean \pm SEM. Comparison of mean values between groups was made using one way ANOVA, and post-hoc analysis was calculated using the Student-Newman-Keuls test. A value of $P<0.05$ was considered significant. Statistical analysis was performed using the statistical package for social studies SPSS version 11.5.

\section{Results}

After UUO, the obstructed kidney was unusually large and often looked paler than the contralateral kidney; when cut open it showed obvious signs of hydronephrosis, containing clear, translucent fluid (Fig. 1B). Representative histology images of the kidneys obtained from SOR and UUO rats are presented in Fig. 3. All morphological lesions observed in the obstructed kidney became more aggravated in time. After 24 hours of UUO, glomerular and tubulointerstitial morphologies were almost intact, and no infiltrating cells were observed. By the end of 72 hours post-obstruction, renal damage was limited to a re- duction of peritubular capillaries, tubular atrophy and widen interstitial space, accompanied by an inflammatory cell infiltration in the interstitium. At 1, 2 and 3 weeks post-obstruction, all lesions observed at earlier time point worsened and increased extracellular matrix (ECM) deposition became a prominent feature. Most tubules appeared markedly atrophic, having lost their original integrity. Atrophic tubules were surrounded by a thickened, wrinkled basement membrane. Glomerular damage was limited to thickening of Bowman's capsule. At 1, 2 and 3 months after UUO, ECM deposition increased further and the interstitial space of the obstructed kidney was populated by numerous fibroblasts. The glomerular size was gradually increased throughout the observation period in the contralateral kidney of UUO rats. By the end of 3 months post-obstruction, focal glomerular sclerosis was present in over one third of glomeruli in the contralateral kidney, and furthermore, tubular atrophy and deformation were also ubiquitous. However, sections derived from the sham kidney and the right kidney of SOR had a normal appearance. 
UUO
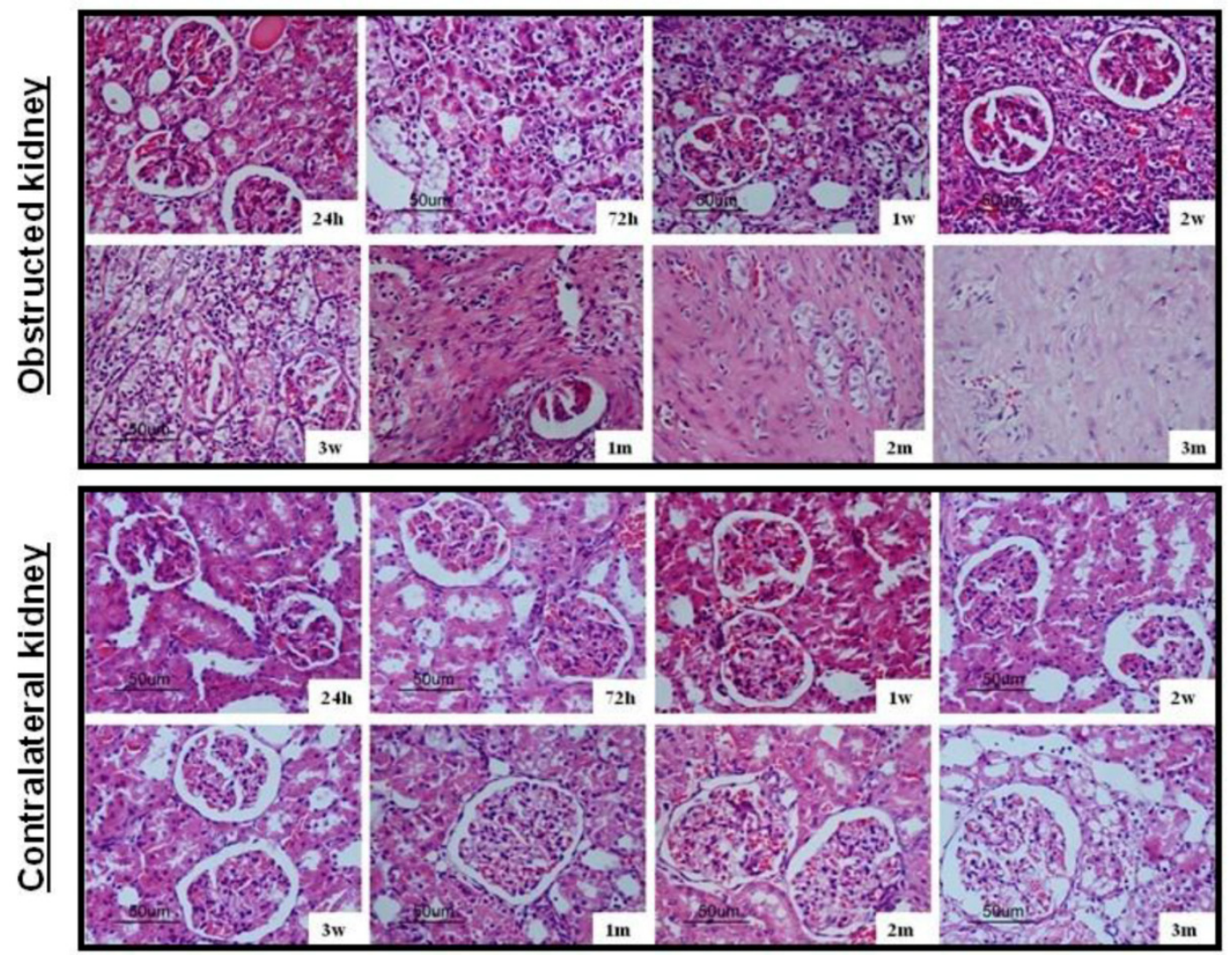

SOR
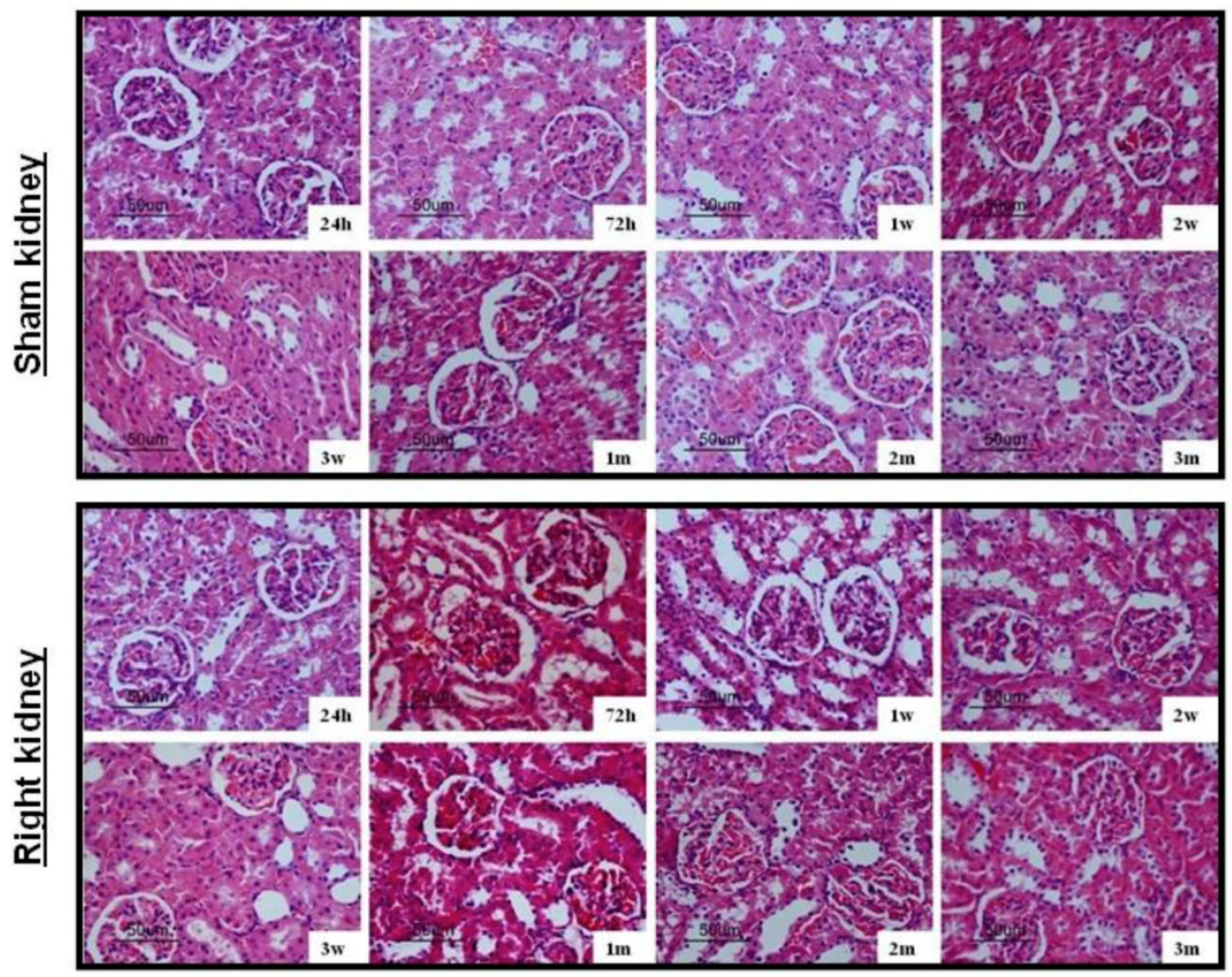

Fig. 3. Representative histology images of the kidneys obtained from SOR and UUO rats. 
TA, GA, TA/RA and GV in bilateral kidneys of UUO rats and SOR are shown in Fig. 4. At the time of sacrifice, TA and TA/RA were raised gradually, but significantly, in the obstructed kidney from 1 to 3 months post-ligation in comparison to the sham kidney of SOR ( $P<0.05$; Fig. 4A and 4C). On the contrary, GA and GV were decreased progressively over time, but significantly, in the obstructed kidney from 3 weeks to 3 months post-ligation compared to the sham kidney of SOR $(P<0.05$; Fig. $4 \mathrm{~B}$ and $4 \mathrm{D})$. UUO caused a progressive decline of TA and TA/RA in the contralateral kidney. More specifically, there were significant decreases in TA at 1, 2, 3 months post-ligation, while in TA/RA only at 3 months post-ligation in comparison to the right kidney of SOR $(P<0.05$; Fig. 4A and 4C). In contrast, GA and GV enhanced in a time-dependent manner in the contralateral kidney, in which the difference in GA reached significance only at 3 months post-ligation, whereas the difference in GV reached significance from 1 to 3 months post-ligation when comparing with the right kidney of SOR $(P<0.05$; Fig. 4B and 4D).
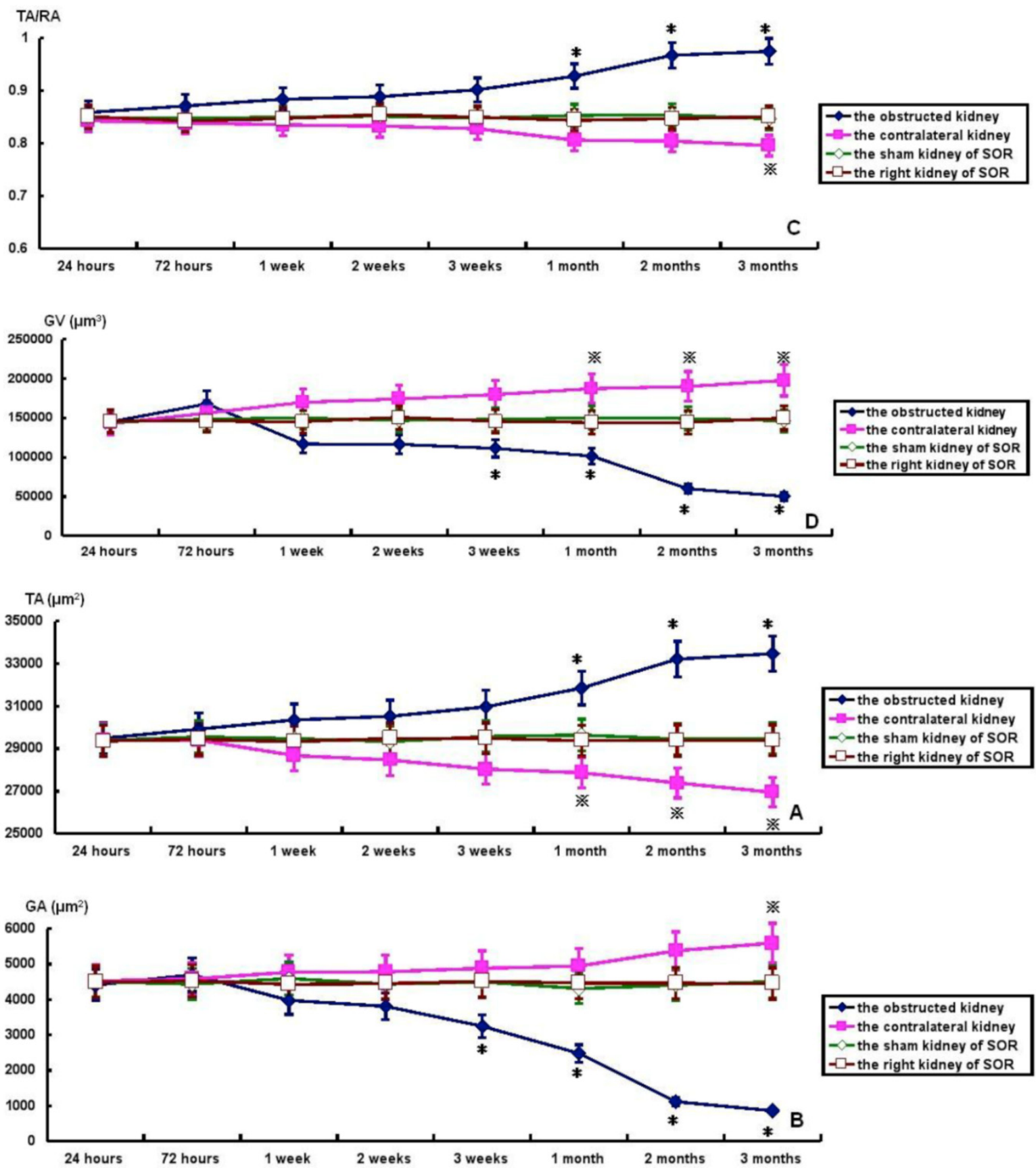

Fig. 4. TA, GA, TA/RA and GV in bilateral kidneys of UUO rats and SOR (Continued). * $P<0.05$, significantly different from the sham kidney of SOR; $※ P<0.05$ compared with the right kidney of SOR. 


\section{Discussion}

Obstructive nephropathy is regarded as a major determinant of progressive renal disease. UUO has been consistently utilized as a convenient model of obstructive nephropathy. The obstructed kidneys were considerably larger and often paler than the contralateral kidneys; when cut open it showed obvious signs of hydronephrosis, containing clear, translucent fluid. In the UUO model, although it becomes more and more apparent that profibrogenic mechanisms are activated very early after the onset of obstruction, apoptosis is also a principal axis throughout the disease course [7]. Our latest study published in Peptides revealed that the ratio of kidney weight to body weight gradually declined in the obstructed kidney during the period from 72 hours to 2 weeks post-ligation, and furthermore, the cortical thickness also decreased time-dependently from 24 hours to 3 months post-ligation [8]. According to the hypothesis of Erkan and Tanaka [9, 10], renal cells subjected to massive proteinuria and prolonged hypoxia-ischemia can induce mitochondrial deficits and persistent energy exhausts, subsequently causing them to undergo apoptosis.

Light microscope observation on histological changes of the obstructed kidney exhibited an obvious periodical variation. Renal damage began to emerge since 72 hours after UUO, characterized by reduced peritubular capillary, notable tubular atrophy, widen interstitial space and inflammatory cell infiltration. When the disease developed into chronic progressive stage, the excessive ECM deposition and numerous fibroblasts became the most prominent feature. The origin of fibroblasts in the kidney is not completely understood, but may derive from resident fibroblasts, from the circulating fibroblast population or from hematopoietic progenitor or stromal cells derived from the bone marrow [11]. In the contralateral kidney, the glomerular size was gradually increased throughout the observation period, which is capable of compensating for the damaged renal unit of the obstructed kidney [4].

In the present study, we established a very simple stereological method to quantify TA, TA/RA, GA and GV. Our results confirmed the previous observations by Karbalay-Doust et al. [12], and found that TA and TA/RA were raised gradually, but significantly, in the obstructed kidney from 1 to 3 months post-ligation in comparison to the sham kidney of SOR; on the contrary, GA and GV were decreased progressively over time, but significantly, in the obstructed kidney from 3 weeks to 3 months post-ligation compared to the sham kidney of SOR.
Because approximately $80 \%$ of the kidney is composed of tubulointerstitium, activated tubulointerstitial cells play a pivotal role in obstructive nephropathy. After UUO, these activated tubulointerstitial cells participate in the initiation of fibrogenic processes which eventually may lead to the elevation in TA and TA/RA [13]. In addition, in the report of Chevalier et al. [14], glomerular size was also significantly reduced in the obstructed kidney, when the persistent ligation exceeded one month. Activation of the renin-angiotensin-aldosterone system may exacerbate glomerular atrophy in the obstructed kidney by constricting the glomerular afferent arterioles, leading to decreased perfusion of the glomerular capillaries [15].

Studies of both animal models and human kidney biopsy specimens have shown that the hypertrophy of the contralateral kidney is another typical feature of obstructive nephropathy. In the present study, GA and GV enhanced in a time-dependent manner in the contralateral kidney, in which the difference in GA reached significance only at 3 months post-ligation, whereas the difference in GV reached significance from 1 to 3 months post-ligation when comparing with the right kidney of SOR. Therefore, the hypertrophy of the contralateral kidney in the UUO model is mainly ascribed to the expanded glomeruli. According to the haemodynamic investigation conducted by Krohn et al. [16], the renal blood flow in the contralateral kidney increased by about $30 \%$ at 5 minutes after UUO. An increased excretory workload is usually postulated to account for the compensatory growth of the contralateral kidney [17]. However, severance of one ureter or its anastomosis into the intestine does not stimulate growth of the contralateral kidney, so it is difficult to attribute the cause of compensatory hypertrophy solely to the excretory overload [18].

In summary, a very simple stereological method was applied in the present study to quantify the renal morphology after UUO and to alleviate the duties of pediatric nephrologists. Our results confirmed two typical features of obstructive nephropathy, including widen interstitial space and glomerular atrophy in the obstructed kidney, and compensatory growth of the contralateral kidney. However, the sensitivity and specificity of this stereological method should be evaluated in the near future.

\section{Acknowledgments}

This study was supported by the National Natural Science Foundation of China (No. 81000306) and the Post-Doctoral Foundation of Anhui Medical University (No. 2009KJ02). 


\section{Competing Interests}

The authors have declared that no competing interest exists.

\section{References}

1. Chevalier RL. Molecular and cellular pathophysiology of obstructive nephropathy. Pediatr Nephrol. 1999; 13(7): 612-619.

2. Hu P, Wang J, Zhao XQ, Hu B, Lu L, Qin YH. Overexpressed C-type natriuretic peptide serves as an early compensatory response to counteract extracellular matrix remodeling in unilateral ureteral obstruction rats. Mol Biol Rep. 2013; 40(2): 1429-1441.

3. Yang J, Dai C, Liu Y. Hepatocyte growth factor gene therapy and angiotensin II blockade synergistically attenuate renal interstitial fibrosis in mice. J Am Soc Nephrol. 2002; 13(10): 2464-2477.

4. Dicker SE, Shirley DG. Compensatory hypertrophy of the contralateral kidney after unilateral ureteral ligation. J Physiol. 1972; 220(1): 199-210.

5. Eisenbrandt DL, Phemister RD. Counting renal corpuscles in tissue sections. Virchows Arch B Cell Pathol Incl Mol Pathol. 1980; 32(2): 177-184.

6. Nyengaard JR. Stereologic methods and their application in kidney research. J Am Soc Nephrol. 1999; 10(5): 1100-1123.

7. Chevalier RL, Thornhill BA, Wolstenholme JT. Renal cellular response to ureteral obstruction: role of maturation and angiotensin II. Am J Physiol. 1999; 277(1 Pt 2): F41-F47.

8. Hu P, Wang J, Hu B, Lu L, Xuan Q, Qin YH. Increased urinary C-type natriuretic peptide excretion may be an early marker of renal tubulointerstitial fibrosis. Peptides. 2012; 37(1): 98-105.

9. Erkan E, Devarajan P, Schwartz GJ. Mitochondria are the major targets in albumin-induced apoptosis in proximal tubule cells. J Am Soc Nephrol. 2007; 18(4): 1199-1208.

10. Tanaka T, Hanafusa N, Ingelfinger JR, Ohse T, Fujita T, Nangaku M. Hypoxia induces apoptosis in SV40-immortalized rat proximal tubular cells through the mitochondrial pathways, devoid of HIF1-mediated upregulation of Bax. Biochem Biophys Res Commun. 2003; 309(1): 222-231.

11. Picard N, Baum O, Vogetseder A, Kaissling B, Le Hir M. Origin of renal myofibroblasts in the model of unilateral ureter obstruction in the rat. Histochem Cell Biol. 2008; 130(1): 141-155.

12. Karbalay-Doust S, Noorafshan A, Pourshahid SM. Taxol and taurine protect the renal tissue of rats after unilateral ureteral obstruction: a stereological survey. Korean J Urol. 2012; 53(5): 360-367.

13. Hodgkins KS, Schnaper HW. Tubulointerstitial injury and the progression of chronic kidney disease. Pediatr Nephrol. 2012; 27(6): 901-909.

14. Chevalier RL, Thornhill BA, Chang AY. Unilateral ureteral obstruction in neonatal rats leads to renal insufficiency in adulthood. Kidney Int. 2000; 58(5): 1987-1995.

15. Hammad FT, Wheatley AM, Davis G. Long-term renal effects of unilateral ureteral obstruction and the role of endothelin. Kidney Int. 2000; 58(1): 242-250.

16. Krohn AG, Peng BB, Antell HI, Stein S, Waterhouse K. Compensatory renal hypertrophy: the role of immediate vascular changes in its production. J Urol. 1970; 103(5): 564-568.

17. Yarger WE, Aynedjian HS, Bank N. A micropuncture study of postobstructive diuresis in the rat. J Clin Invest. 1972; 51(3): 625-637.

18. Sidoti O, Lupo A, Comunale L, Loschiavo C, Luzzani A. Renal lesions after unilateral ureterosigmoidostomy in the rabbit: preliminary results. Urol Res. 1981; 9(3): 123-131. 